\title{
Solution to doubling spectroscopy challenge
}

\section{Reinhard Meusinger ${ }^{1}$}

(C) Springer-Verlag Berlin Heidelberg 2016

The winner of the doubling spectroscopy challenge (published in volume 407, issue 27) is:

Sami T. Tuomivaara, Complex Carbohydrate Research Center, University of Georgia, Athens, GA, USA

The award entitles the winner to select a Springer book of his choice up to a value of $€ 100,-$.

Our Congratulations!

\section{Solution}

The reason for the signal doubling of the H-6 hydrogen signal in the ${ }^{1} \mathrm{H}-\mathrm{NMR}$ spectrum of dexelvucitabine (DFC) is the presence of the fluorine at position 5 (Fig. 1).

Fluorine has only one isotope, ${ }^{19} \mathrm{~F}$, with a spin $1 / 2$ nucleus. Since both ${ }^{1} \mathrm{H}-6$ and ${ }^{19} \mathrm{~F}-5$ are connected in DFC by a vicinal coupling ${ }^{3} J_{\mathrm{H} 6, \mathrm{~F} 5}$, the NMR signal of each ${ }^{1} \mathrm{H}-6$ nucleus is split by the interaction with the ${ }^{19} \mathrm{~F}-5$ nucleus and vice versa. Therefore, no cross-peak will be observed for the H-6 doublet in the H,H-COSY spectrum. As expected, noticeable crosspeaks were observed in the NOESY spectrum for interactions between $\mathrm{H}-6, \mathrm{H}-2^{\prime}, \mathrm{H}-5^{\prime}$, and $-\mathrm{OH}$ protons, indicating correct signal assignment (Fig. 2).

Considerable cross-peaks were also observed between the pair of twin amino proton signals in the NOESY spectrum.

This article is the solution to the Analytical Challenge to be found at http://dx.doi.org/10.1007/s00216-015-9002-x

Reinhard Meusinger

meusi@oc.chemie.tu-darmstadt.de

Institute of Organic Chemistry and Biochemistry, University of Technology Darmstadt, Alarich-Weiss-Str. 4,

64287 Darmstadt, Germany
However, the phase of these cross-peaks (red color) is opposite to that of the other NOESY cross-peaks (blue color). This indicates that there is a slow exchange process between both amino protons during the 700 -ms continuous mixing time at room temperature.

The energy barrier for this exchange process can be calculated using the experimentally determined coalescence temperature $T_{\mathrm{c}}$ and the frequency difference $\Delta \nu$ between the separate signals of the amino-group protons. Both of these parameters were determined twice at different magnetic field strengths:

$$
\begin{array}{ll}
T_{\mathrm{c}, 300 \mathrm{MHz}}=314 \mathrm{~K} & T_{\mathrm{c}, 500 \mathrm{MHz}}=322 \mathrm{~K} \\
\Delta \nu_{300 \mathrm{MHz}}=73.5 \mathrm{~Hz} & \Delta \nu_{500 \mathrm{MHz}}=123 \mathrm{~Hz}
\end{array}
$$

By using the Boltzmann constant $k_{\mathrm{B}}$, the Planck constant $h$, and the universal gas constant $R$, the

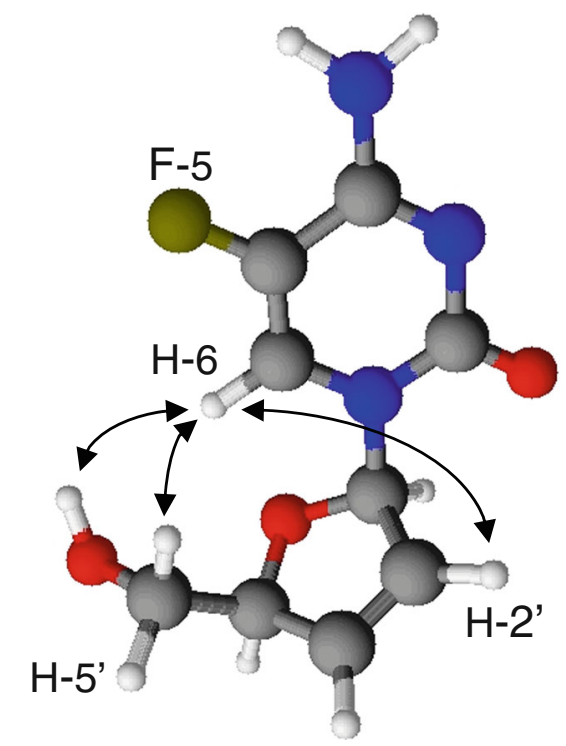

Fig. 1 Three-dimensional structure of DFC with observed NOEs for proton H-6 (black arrows) 
Fig. 2 Two-dimensional ${ }^{1} \mathrm{H}$ correlated double quantum filtered COSY spectrum (left) and NOESY spectrum (right) of DFC. The phase of the NOE crosspeaks (blue) is opposite to that of the diagonal peaks $(\mathrm{red})$. The redcolored cross-peaks between both amino protons relate to the intramolecular exchange process

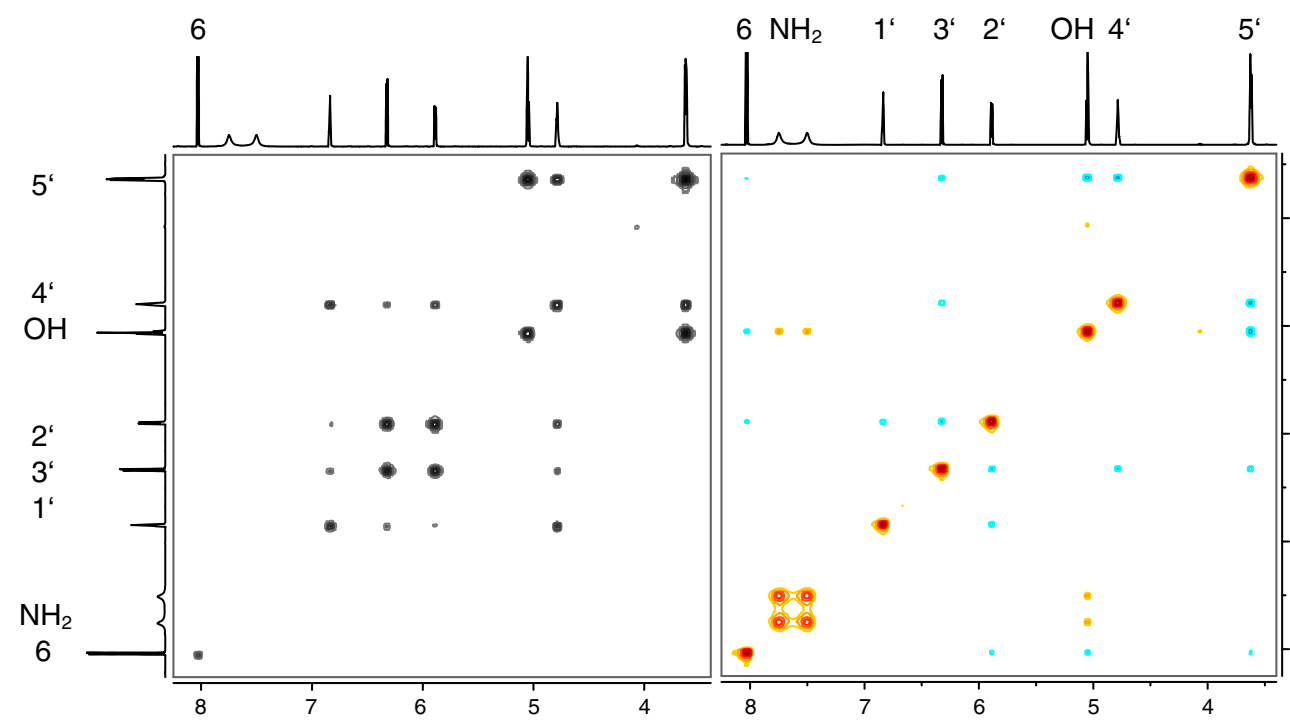

This yields $\Delta G_{300 \mathrm{MHz}}^{\#}=63.7 \mathrm{~kJ} \mathrm{~mol}^{-1}$ and $\Delta G_{500 \mathrm{MHz}}^{\#}=64.0 \mathrm{~kJ} \mathrm{~mol}^{-1}$.

Accounting for small experimental deviations, a very similar result of $64 \mathrm{~kJ} \mathrm{~mol}^{-1}$ was obtained in both experiments. 